\title{
The Effects of Matched Filter on Stable Performance of Semistrapdown Inertially Stabilized Platform
}

\author{
Feng Liu and Hua Wang \\ School of Astronautics, Beihang University (BUAA), Beijing 100191, China \\ Correspondence should be addressed to Feng Liu; lfjssx@126.com
}

Received 4 March 2016; Revised 2 May 2016; Accepted 26 June 2016

Academic Editor: Romain Aubry

Copyright (C) 2016 F. Liu and H. Wang. This is an open access article distributed under the Creative Commons Attribution License, which permits unrestricted use, distribution, and reproduction in any medium, provided the original work is properly cited.

\begin{abstract}
To enhance the optimization performance of matched filter and further improve line of sight (LOS) stability of platform in inertial space, the proposed matched filter algorithm is conducted by adjusting matched filter coefficients of first-order low pass filter utilizing the regional search method based on invariance principle. The coefficients of the fraction molecule and denominator of proposed regional search algorithm are altered instead of denominator coefficients only being modified. Simulations are performed to verify the validity of inside factors performed with stabilization control model and quartz rate sensor (QRS) mathematical model. The stable angular error is sharply alleviated, so the decoupling accuracy of airborne semistrapdown inertially stabilized platform is largely promoted. The optimization matched filter can effectively increase stability of LOS in inertial space.
\end{abstract}

\section{Introduction}

Photoelectric sensor is a device of great importance for detecting and tracking targets in semistrapdown stabilized platform [1]. LOS of the photoelectric sensor is affected when flying body attitude changes because the photoelectric sensor is directly connected with flying body in [2], which leads to unstable optical axis and nonideal tracking effect. The noise of sensor is attenuated using matched filter in general. The rate gyro sensor can measure azimuth and pitch angular velocity. While the angular velocity is directly fed back to actuator and makes LOS reversely deflect so as to achieve stabilization, the attitude information is fed back to the closed loop according to space coordinate transformation when the information is measured by inertia devices of flying bodies, which make frames reduce vibration by flying bodies disturbance.

Scholars put forward some opinions on increasing stability. In 1993, strapdown platform model was investigated and decoupling results of QRS and FOG sensor were obtained in [3]. In recent years, a controller was established based on offline initialization to get the optimal controller and modeling errors were solved by optimization filters in [4]. The stability of parasitic loop induced by disturbance rejection effect (DRE) of a semistrapdown homing seeker (SSHS) was employed in [5]. The sensors' dynamic errors of strapdown detector and rate gyro based on guidance system were addressed in [6]. The matching of rate gyro and dynamics were researched utilizing constraining nonlinear minimization optimization method in [7]. A newly continuously differentiable friction model and filtered regression estimation parameter were introduced; the stability of the proposed methods was proved in [8]. The matched filter was expressed in order to suppress and compensate the imperfect influence of nonlinear friction force factors in [9-11], for instance, the static friction force of the frame and motor dead zone phenomenon.

Previous matched filter researches are just mostly focused on the change of the denominator coefficient, while the molecular coefficient of the first-order low-pass filter is a fixed parameter. However, to further improve the optimization performance, the proposed regional search algorithm dynamically limits the search area and reduces the search complexity of the algorithm in time and space, so the operation efficiency of the algorithm is greatly improved.

The overall paper is organized as follows: Section 1 addresses the research purpose; Section 2 presents the control 


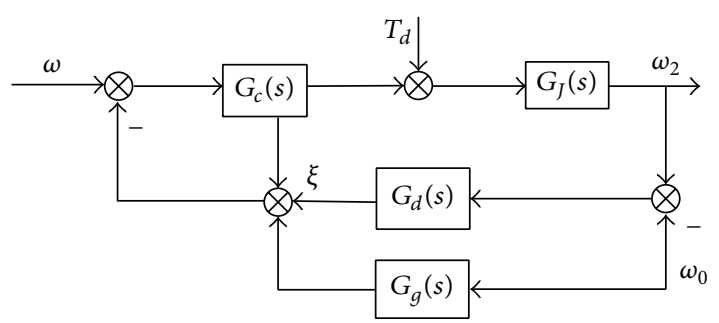

FIGURE 1: Control model of semistrapdown platform.

model of semistrapdown stabilization; Section 3 develops matched filter optimization algorithms; Section 4 proves the efficiency of the proposed matched filter optimization algorithm; and Section 5 summarizes theoretical and practical engineering significance of the study.

\section{Semistrapdown Stabilization Control Model}

2.1. Stabilization Principle. The two-axis and two-frame miniature semistrapdown stabilization platform has its advantages, which makes it become a great tool for the integration of investigation and combat. In [12], the mathematical model of semistrapdown stabilization control is shown in Figure 1.

In this system, $\omega$ is angular velocity instruction, $\omega_{2}$ is angular velocity of stabilized platform under inertial axis, flying bodies attitude disturbance angular velocity is $\omega_{0}, \eta$ denotes measurement noise, $T_{d}$ is disturbance torque, $G_{J}$ is control object transfer function, $G_{c}$ is transfer function of speed loop controller in [13], $G_{d}$ represents transfer function of measurement rate link, and $G_{g}$ represents gyro transfer function. The control model of semistrapdown platform is shown in Figure 1. The angular velocity of semistrapdown stabilized platform control model under inertial axis is extracted as

$$
=\frac{G_{J} \cdot G_{c} \cdot \omega+G_{J} \cdot G_{c} \cdot\left(G_{d}-G_{g}\right) \cdot \omega_{0}+G_{J} \cdot T_{d}-G_{J} \cdot G_{c} \cdot \eta}{1+G_{J} \cdot G_{c} \cdot G_{d}}
$$

where $\omega_{0}=A \sin (2 \pi f t), f$ is body disturbance frequency, and $A$ denotes the maximum amplitude of the angular velocity of the flying bodies.

When $\omega, T_{d}$, and $\xi$ are ignored, we have

$$
\omega_{2}=\frac{G_{J} \cdot G_{c}\left(G_{d}-G_{g}\right)}{1+G_{J} \cdot G_{c} \cdot G_{d}} \omega_{0} .
$$

The disturbance of body to the LOS is eliminated when $G_{g}=G_{d}$ from (2), which is ideally equivalent to the complete decoupling of semistrapdown stabilization.

2.2. Simplified Control Model. The closed-loop structure of stable rate is simplified as shown in Figure 2.

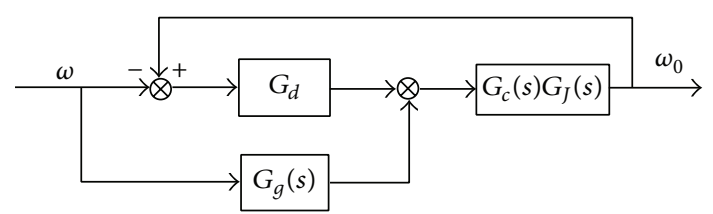

FIGURE 2: The closed-loop structure of stable rate.

TABLE 1: The related data of the QRS model.

\begin{tabular}{lc}
\hline Dynamic component & Expression \\
\hline Compensation & $\frac{700(s / 50+1)}{s(s / 1000+1)}$ \\
Inertia & 0.05 \\
Differentiator & $\frac{s}{(s / 7560+1)}$ \\
Gyro & $\frac{1}{s^{2} / 1.9455 e 5+s / 315.46+1}$ \\
Converter & $(s / 18850) \cdot\left(s^{2} / 3.578 e 8+s / 13398+1\right)$ \\
\hline
\end{tabular}

\section{The Optimization of Matched Filter Algorithm}

In order to make $G_{g}=G_{d}$, matched filter algorithm is proposed in engineering, as shown in (2), where $G_{d}$ is conducted under the matched filter according to invariance principle. The transfer function of matched filter is assumed to be

$$
G=\frac{a \cdot s+1}{b \cdot s+1}, \quad(a<b) .
$$

The related data of the QRS model of matched filter algorithm is based on [3], as reflected in Table 1.

3.1. Algorithm 1. $G_{0}$ represents the transfer function before matched filter (matched filter is not used), which can be expressed as

$$
G_{0}=\frac{\omega_{0}}{\omega_{1}}=\frac{\left(G_{d} \cdot G_{R / D}-G_{g}\right) \cdot G_{c} \cdot G_{J}}{1+G_{d} \cdot G_{R / D} \cdot G_{c} \cdot G_{J}} .
$$

The transfer function after matched filter is

$$
G_{1}=\frac{\omega_{0}}{\omega_{1}}=\frac{\left(G_{d} \cdot G_{R / D} \cdot G_{m}-G_{g}\right) \cdot G_{c} \cdot G_{J}}{1+G_{d} \cdot G_{R / D} \cdot G_{m} \cdot G_{c} \cdot G_{J}} .
$$

The objective function can be established by the nonlinear constrained optimization algorithm of mechanical optimization design scheme [14]:

$$
f(x)=\min \left(\left|G_{1}\right|-\left|G_{0}\right|\right),
$$

where the constraint condition is $a \geq b \geq 0$. At last, $a=$ $0, b=0.03$ is obtained by simulation.

3.2. Algorithm 2. The flowchart of Algorithm 2 is indicated in Figure 3.

(i) The First Step. In order to compare with Algorithm 1, the interval $[0,0.01]$ is regarded as research subject. The interval 


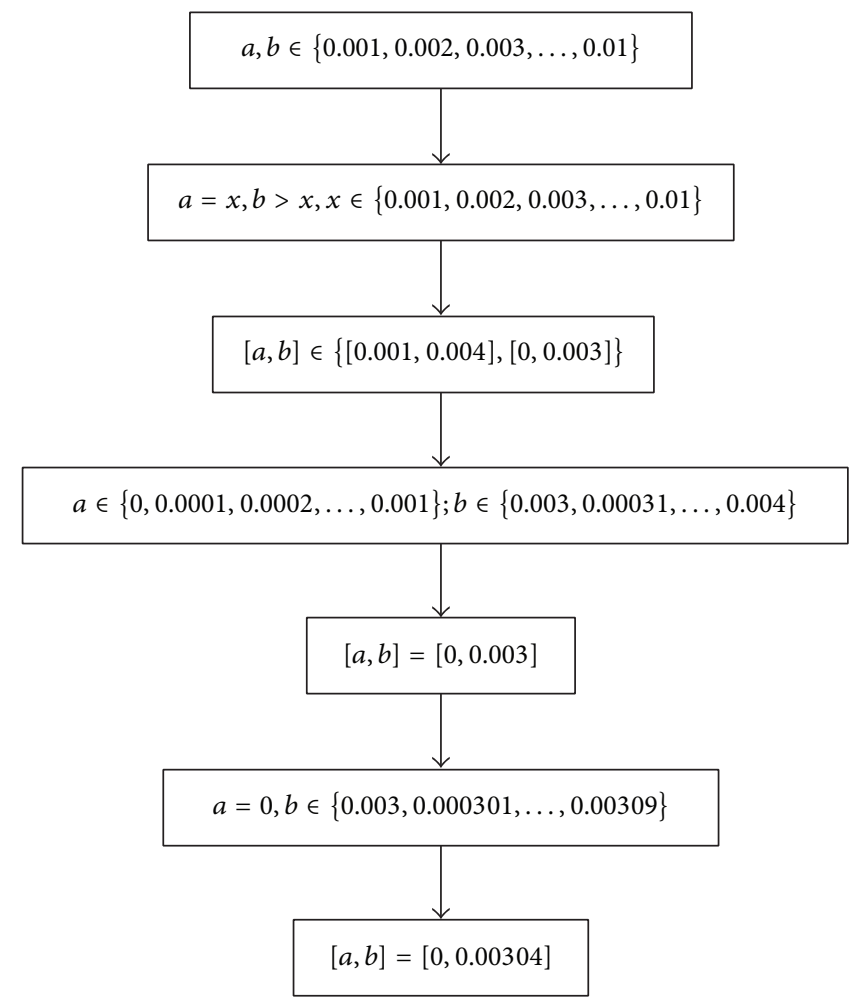

FIGURE 3: Flowchart of Algorithm 2.

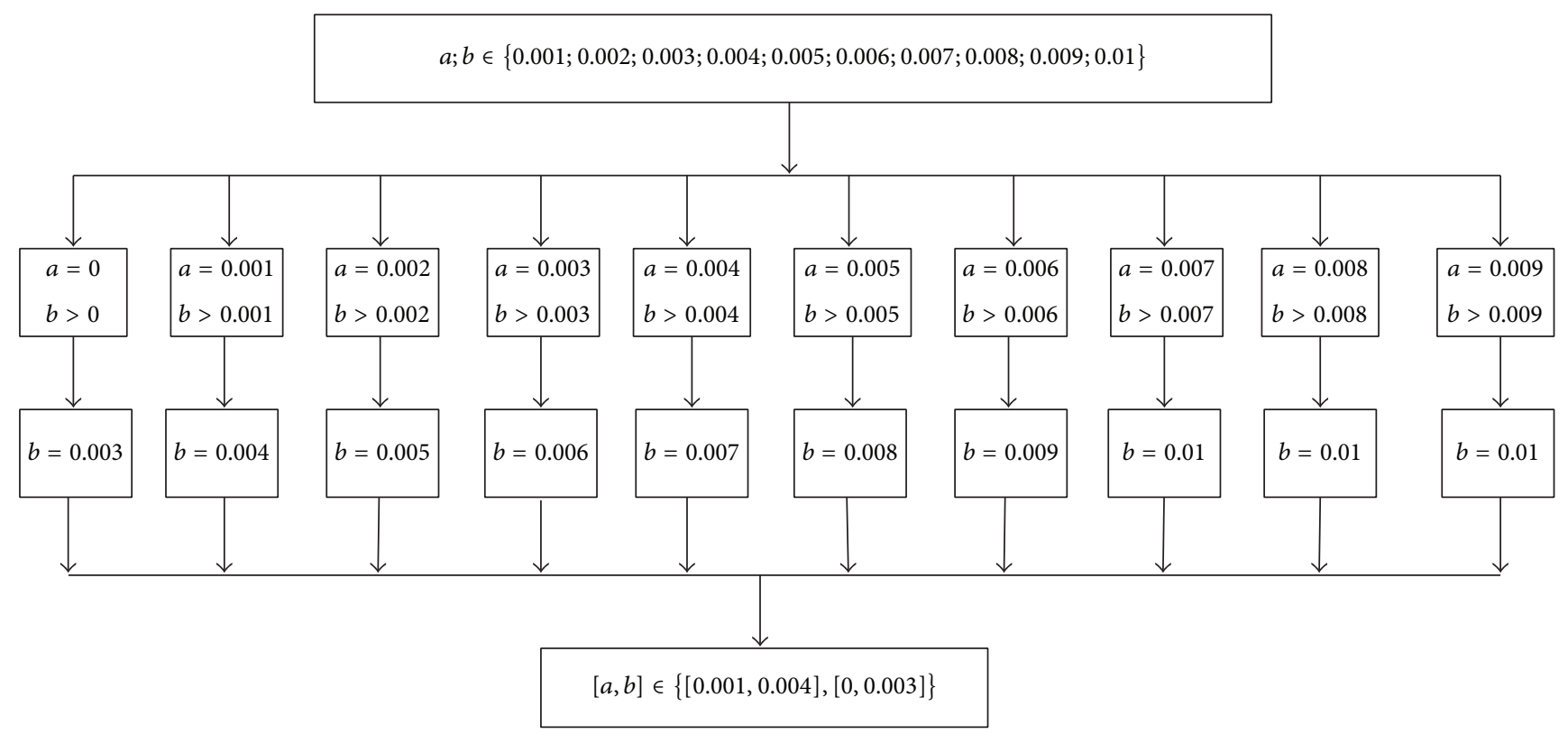

FIGURE 4: Algorithm structure diagram of the first step.

$[0,0.01]$ is divided into 10 equal parts; there are eleven numbers from $0,0.001$ to 0.01 . Let $a, b \in\{0,0.001,0.002, \ldots, 0.01\}$; 10 kinds of situations are displayed in line two of Figure 4 when $b$ is greater than $a$. The optimal matching of every situation is acquired by simulation. Then, the best decoupling characteristics are regarded as a new group; then, 10 groups are reflected in line 3 of Figure 4 . Followed by analogy, the better of two groups is shown in Figure 4.
The better simulation effect of $[0.001,0.004]$ and $[0,0.003]$ is obtained from Figure 4 ; then, the better result is searched from $[0,0.001]$ and $[0.003,0.004]$.

(ii) The Second Step. The interval $[0,0.001]$ is divided into 10 equal parts; there are eleven numbers from $0,0.0001$ to 0.001 . Let $a \in\{0,0.001,0.002, \ldots, 0.01\}$. Similarly, the interval $[0.003,0.004]$ is divided into 10 equal parts, where 


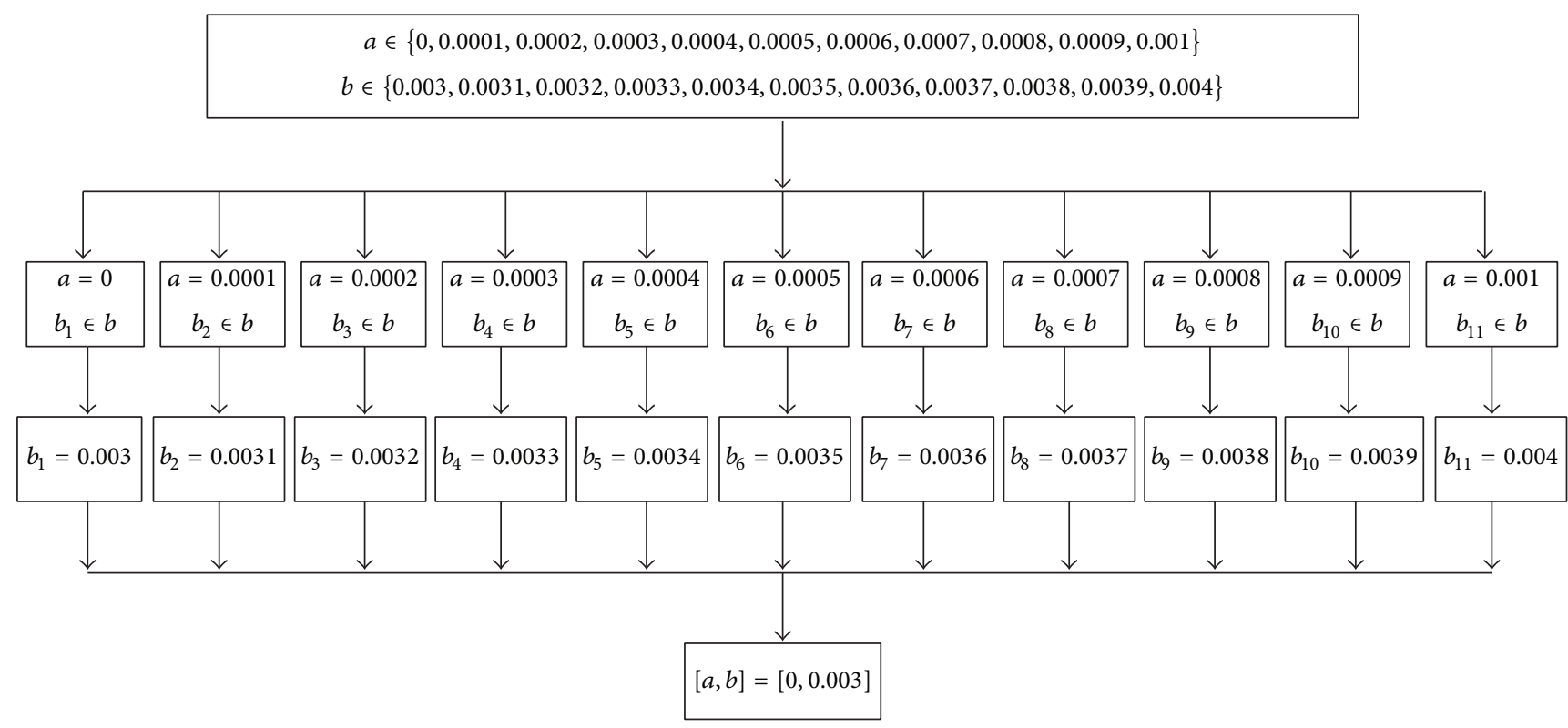

FIGURE 5: Algorithm structure diagram of the second step.

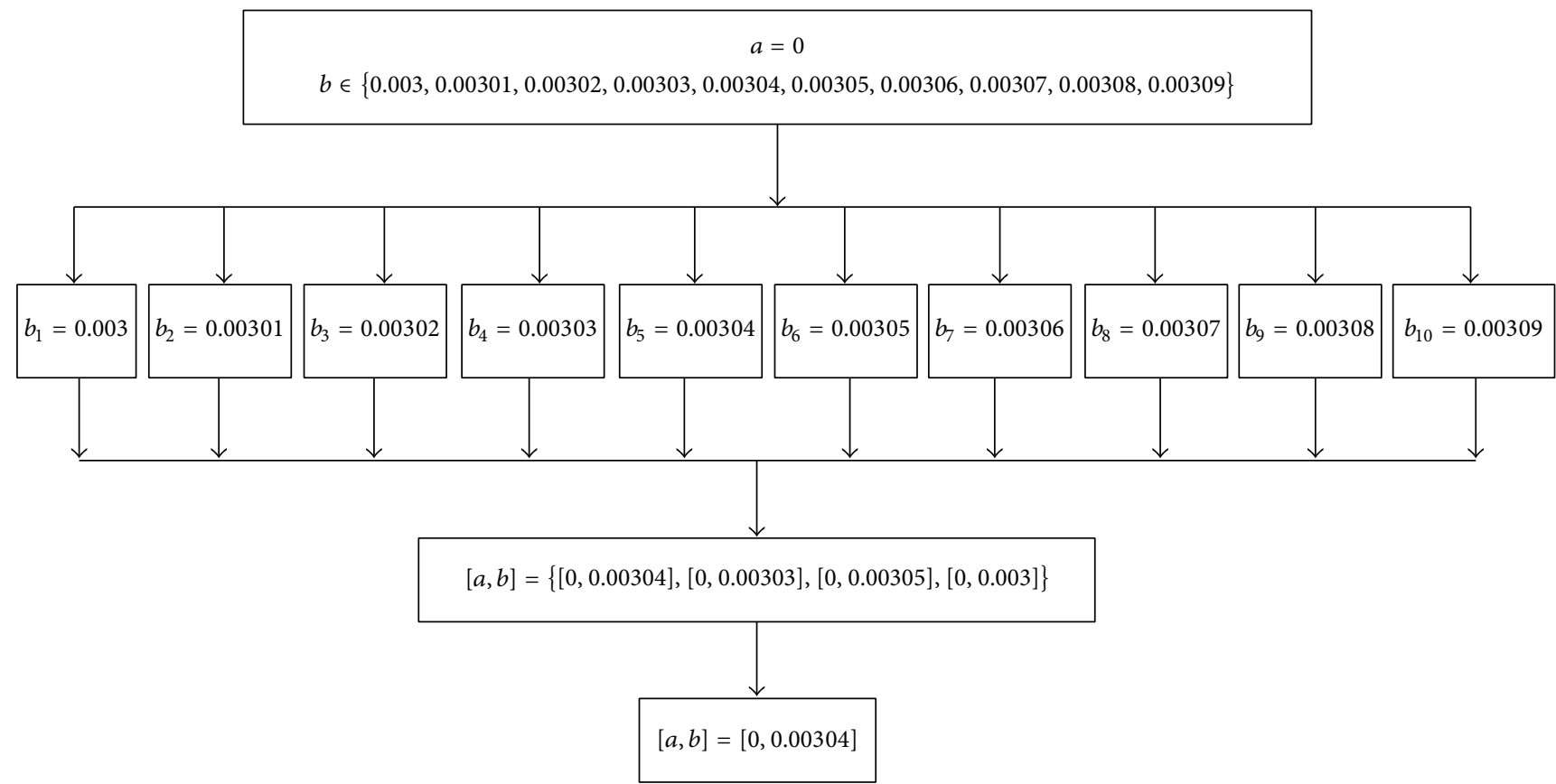

FIgURE 6: Algorithm structure diagram of the third step.

there are eleven numbers from $0.003,0.0031$ to 0.004 . Let $b \in\{0.003,0.0031, \ldots, 0.004\}$; thus, eleven kinds of situations are illustrated in line two of Figure 5 when $b_{i} \in b, i=$ $1,2, \ldots, 11$. The optimal matching of every situation is gained by simulation. Then, the best decoupling characteristics are regarded as a new group, and 11 groups are reflected in line 3 of Figure 5. Followed by analogy, the best group is shown in Figure 5.
The last result of Figure 5 is completely consistent with the result of Algorithm 1 by simulink. However, we hope to find a better result by search method.

(iii) The Third Step. Let $b \in\{0.003,0.00301,0.00302, \ldots$, $0.00309\}$ and $a=0$. Ten kinds of situations are shown in line 2 of Figure 6 . Then, four groups of the better decoupling characteristics are selected; they are reflected in line 3 of Figure 6. Followed by analogy, the best group is shown in 


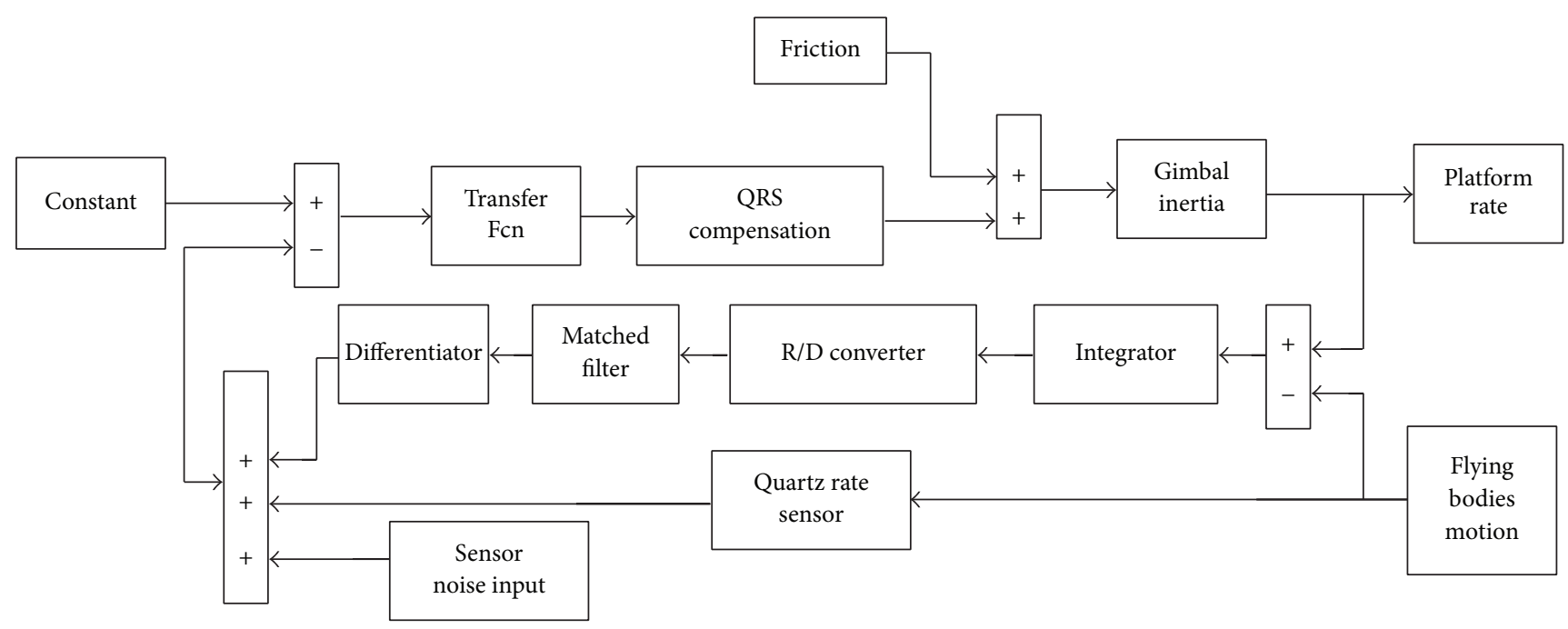

FIgURE 7: Optimization model of inertial stabilization platform.

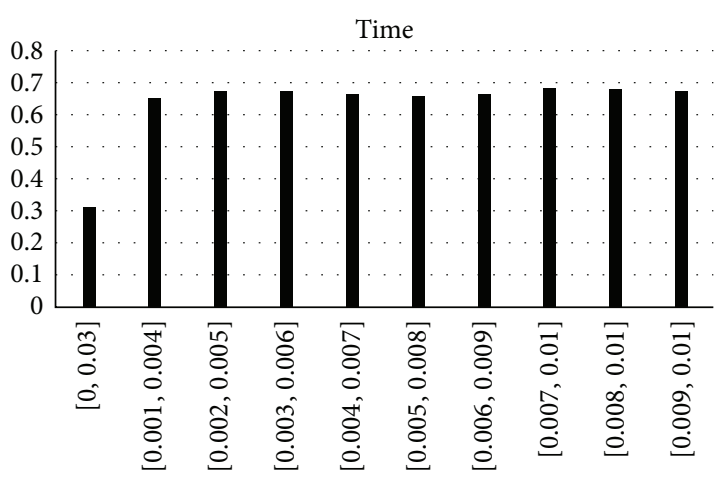

(a)

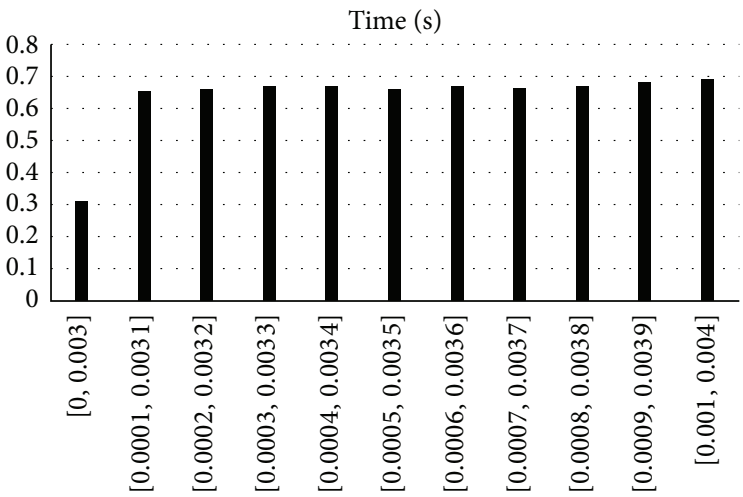

(b)

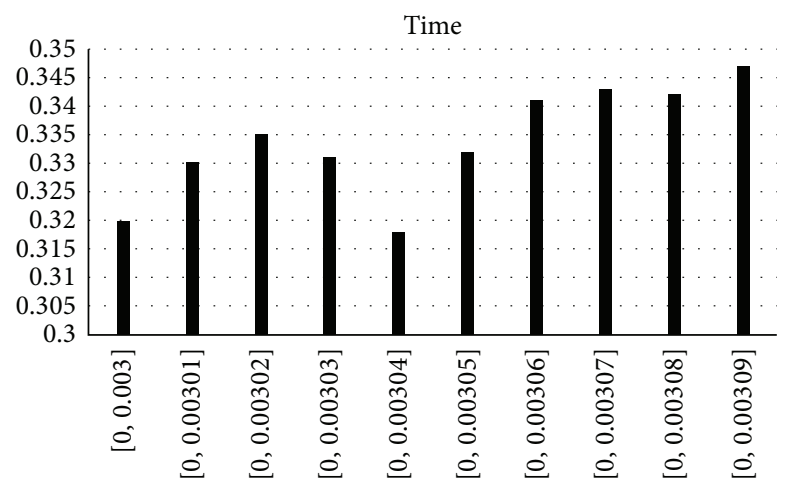

(c)

FIGURE 8: Step response time of three steps.

Figure 6. The last result of Figure 6 is a perfect result by simulink.

\section{Validation Test and Simulation Analysis}

In order to better explain the validity of the algorithm, taking the closed-loop stability control system into consideration, the simulation model in [15] is shown in Figure 7.

\subsection{Simulation Experiment Validations}

4.1.1. The Step Simulation Experiments. Considering the speed of reaching the steady state of the system, the step response is presented in Figure 8.

The time of reaching the steady state is very principal for engineering application. It is clear that the $[0.001,0.004]$ and $[0,0.003]$ are excellent among ten group coefficients of the 


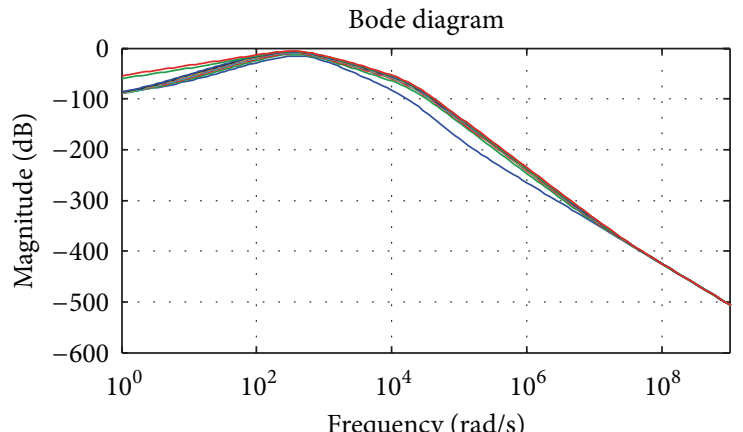

$[0,0.003]$

$-[0.001,0.004] \quad[0.006,0.009]$

$-[0.002,0.005] \quad-[0.007,0.01]$

$-[0.003,0.006]-[0.008,0.01]$

$-[0.004,0.007] \quad-[0.009,0.01]$

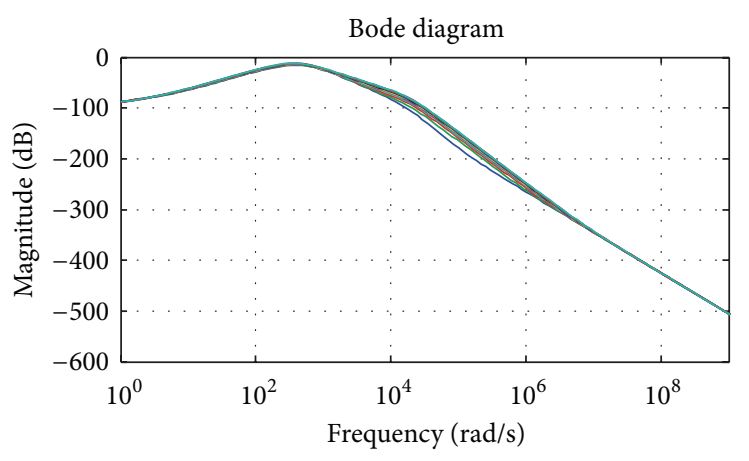

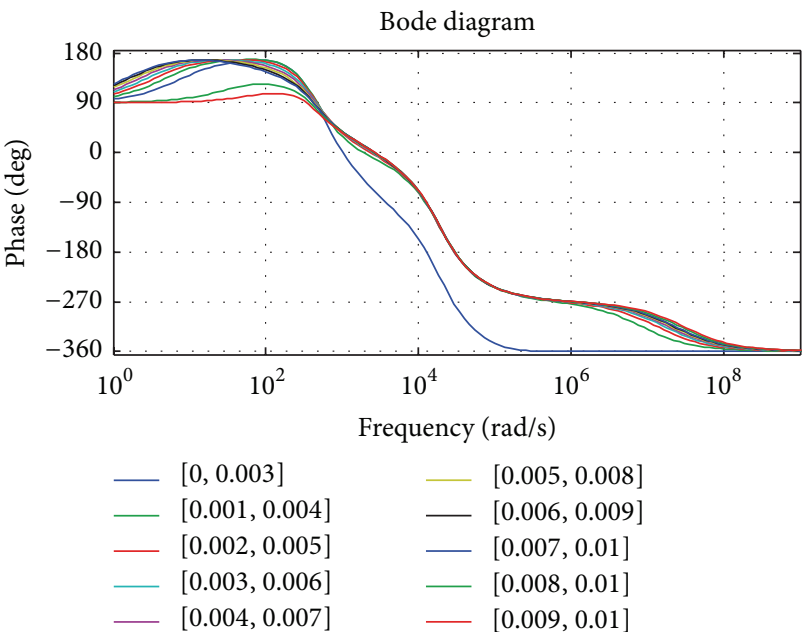

(a)

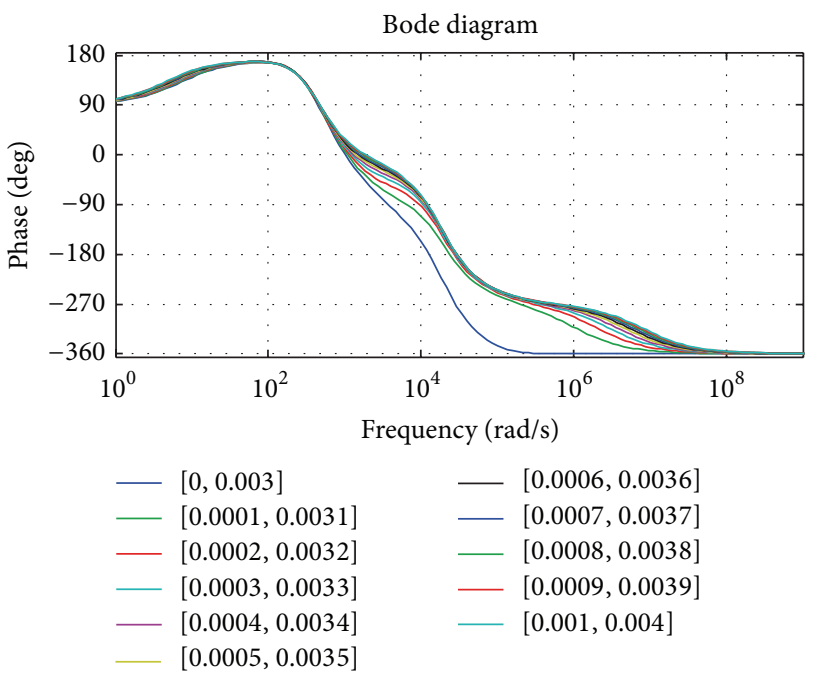

(b)

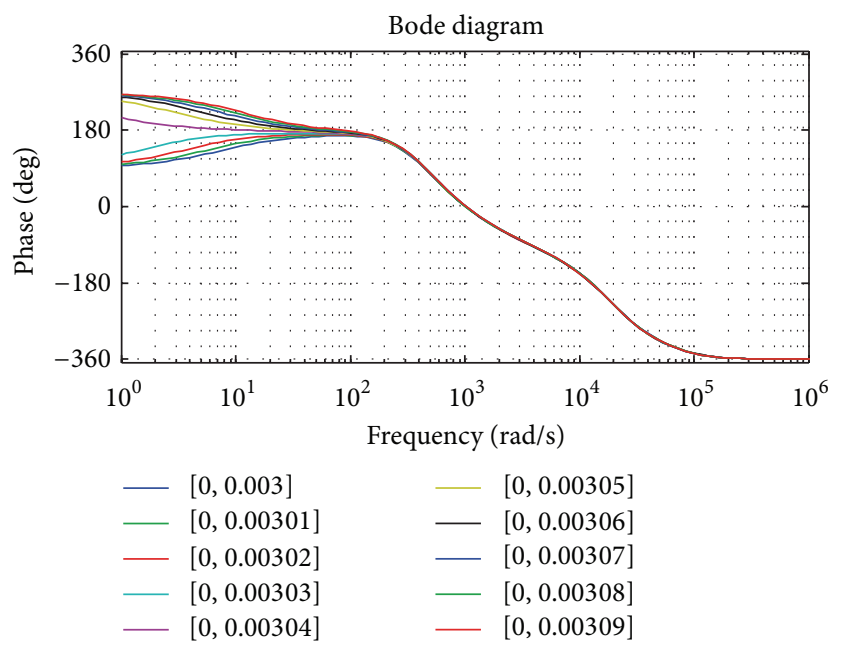

(c)

FIGURE 9: Comparison of decoupling accuracy of four groups' matched filter. 

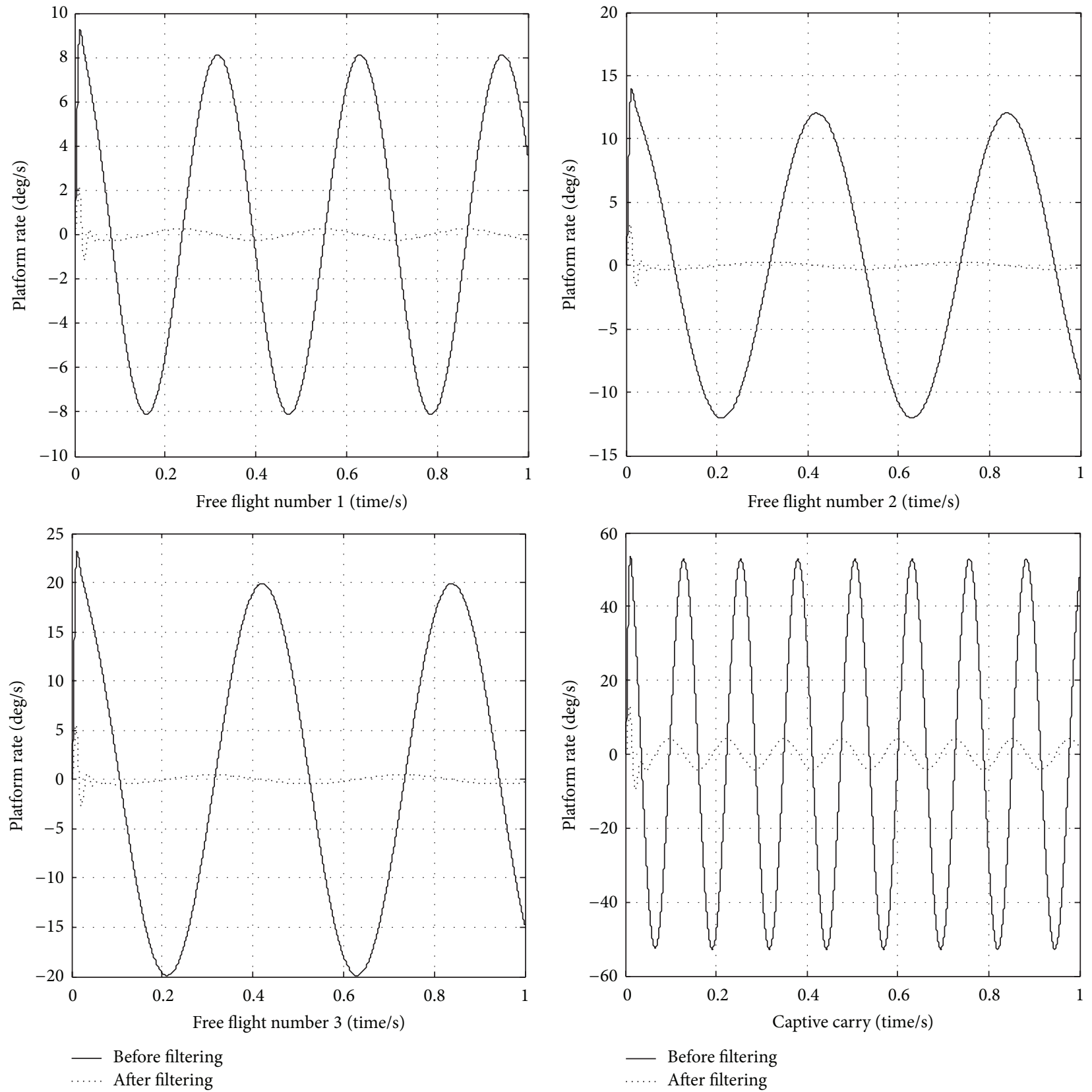

FIGURE 10: The platform rate before matched filter and after matched filter.

first step in Figure 8(a); the result of simulation experiments is consistent with the result of Algorithm 1, and the time of $[0.001,0.004]$ and $[0,0.003]$ when arriving at the steady state is shorter than others. The step response is indicated in Figure 8(b); the reaching speed of the steady state about the coefficient $[0,0.003]$ is faster than others. The coefficient of $[0,0.00304]$ is the best among ten coefficients, and it can be confirmed based on Figure 8(c).

4.1.2. Bode Diagram Simulation Experiments. The matching effect of the matched filter plays an indispensable role in engineering. It is helpful even if there is a little improvement, as is shown in Figure 9.

The coefficients $[0.001,0.004]$ and $[0,0.003]$ of Figure $8(a)$ are very prominent. The coefficient $[0,0.003]$ of the second step is indicated in Figure 8(b), where it is shown that the optimization result is in accordance with the result of Algorithm 1. The coefficients [0, 0.00304], [0, 0.00303], $[0,0.00305]$, and $[0,0.003]$ are better from Bode diagram of the closed-loop control simulation. But the coefficient $[0,0.00304]$ is the best. Figure 8 (c) can be illustrated by this truth. Meanwhile, the coefficient $[0,0.00304]$ has a 
TABLE 2: The input rate of flying bodies.

\begin{tabular}{lcc}
\hline Environment & \multicolumn{3}{c}{ Rate $\cdot \omega_{m}$} \\
\hline Free flight number 1 & Deg/s & $\mathrm{Hz}$ \\
Free flight number 2 & 130 & 5 \\
Free flight number 3 & 260 & 2.5 \\
Captive carry & 430 & 5 \\
\hline
\end{tabular}

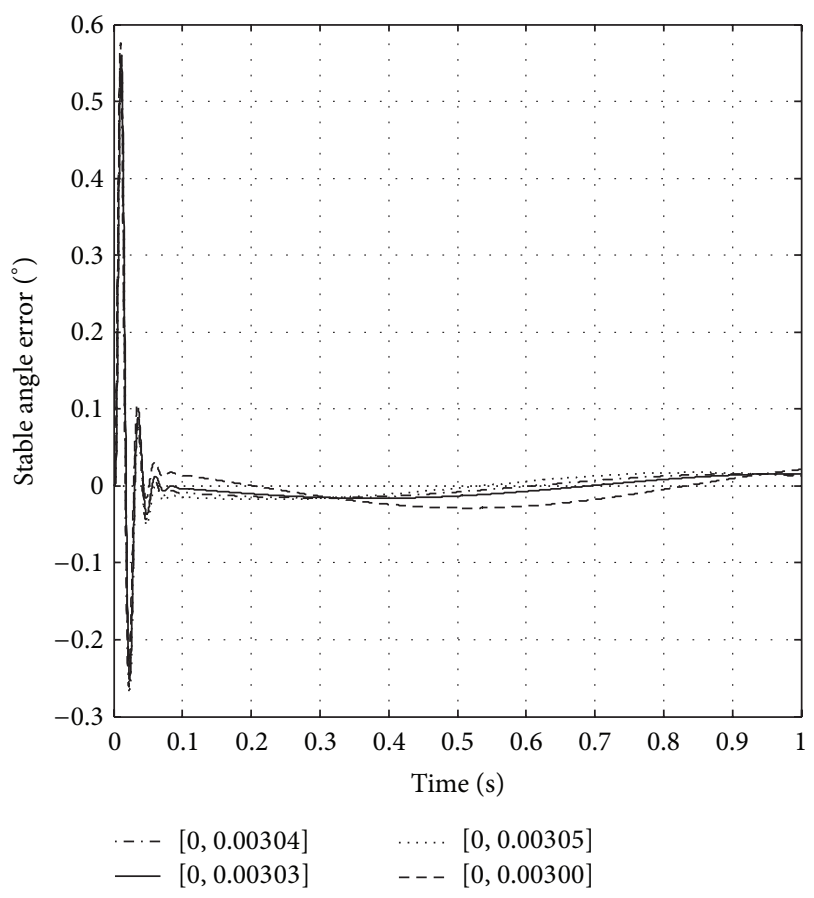

FIGURE 11: Stable angle error under different matched filter.

relatively higher decoupling accuracy, and the noise also can be decreased, so $G_{m}=1 /(0.00304 s+1)$ is the best matched filter.

\subsection{Stable Error before Matched Filter and after Matched Filter}

4.2.1. The Rate Comparison of Platform before and after Matched Filter. The related data of flying bodies angular velocity motion is given based on [3], which is used as the verification test when the input signal is the unit step signal, as shown in Table 2.

As shown in Figure 10, the rate of stable platform before matched filter and after matched filter is as follows.

Simulation results from Figure 10 show that the angular rate of the platform is declined by $90 \%$, so the optimization results are very good.

\subsubsection{The Comparison of Stable Angle Error under Different} Matched Filter. The stable angle errors of the four groups' matched filter of Figure 8 are compared utilizing search method, and their differences are revealed in Figure 11.

Stable angle error is obviously distinct using dissimilar matched filter from Figure 11; the solid line stands for the best matched filter; its error of the stable angle is smaller.

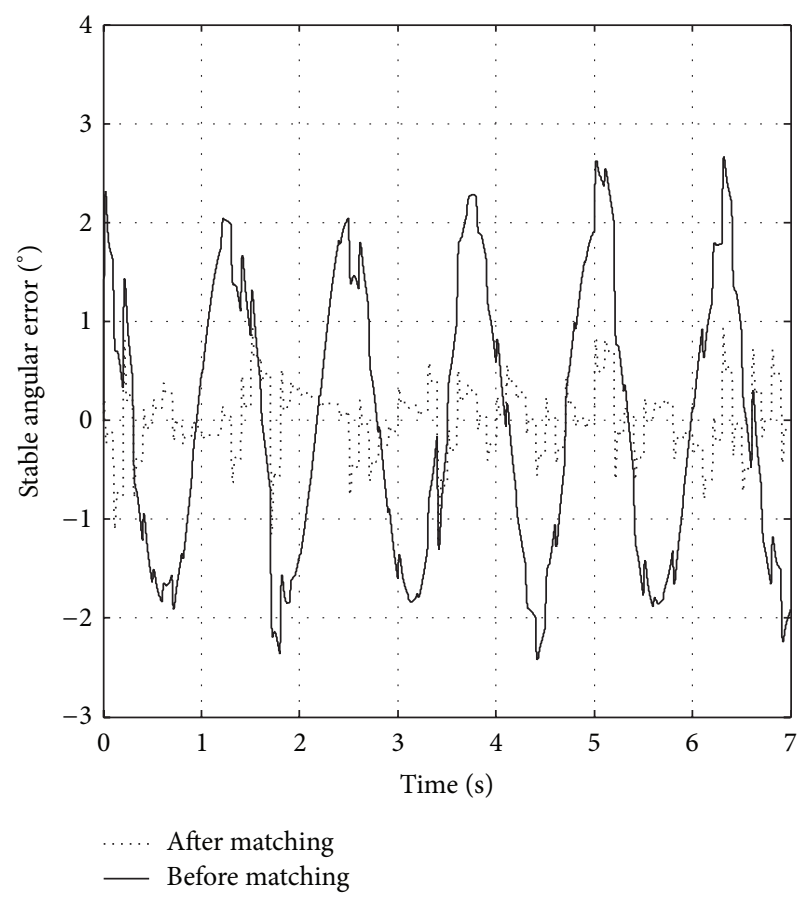

FIGURE 12: Stable angular error.

4.3. Effect on the Stable Error of Sensor Measurement Noise. The angular velocity of the semistrapdown stabilized platform is obtained by rate gyroscope and related calculation from Figure 1 and (1). The measurement noise of the sensor has influence on stabilization of semistrapdown stabilized platform. The amplification factor of the measurement rate can be enlarged, but not without limitation. The matched filter algorithm is proposed in order to reduce the noise in engineering.

In Figure 12, the measurement noise of the sensor has a great effect on the stability of the angle error, and the stability of the angle error is diminished by nearly $70 \%$ after matched filter.

\section{Conclusions}

(1) Measurement rate is matched by first-order low pass filter based on invariance principle. Simulations show that the angular rate of the platform is lessened by $90 \%$ after matched filter. Not only can we get the result of Algorithm 1, but also we can obtain The optimal matching, which can promote decoupling accuracy as far as possible.

(2) The measurement noise of sensor has huge influence on the stable error. The stability of the angle error is decreased by nearly $70 \%$ after matched filter.

(3) The stability of LOS can be strengthened based on the above simulation results. So it provides theoretical foundations for designing and optimization of the microstable platform, which has a strong guiding significance in engineering. 


\section{Competing Interests}

The authors declare that there is no conflict of interests regarding the publication of this paper.

\section{References}

[1] S. Yin, H. Jia, Y. Zhang, and H. Gao, "Semi-strapdown stabilization of optical imaging seeker," Infrared and Laser Engineering, vol. 40, no. 1, pp. 129-148, 2011.

[2] X. Zhou, Z. Zhang, and D. Fan, "Improved angular velocity estimation using MEMS sensors with applications in miniature inertially stabilized platforms," Chinese Journal of Aeronautics, vol. 24, no. 5, pp. 648-656, 2011.

[3] R. T. Rudin, "Strapdown stabilization for imaging seekers," in Proceedings of the 2nd Annual AIAA SDIO Interceptor Technology Conference, pp. 1-10, June 1993.

[4] Z.-R. Tsai, "Neural-fuzzy digital strategy of continuous-time nonlinear systems using adaptive prediction and random-localoptimization design," Mathematical Problems in Engineering, vol. 2013, Article ID 836414, 12 pages, 2013.

[5] S. Jianmei, C. Gaohua, C. Xianxiang, and K. Lixia, "Stability region analysis of the parasitic loop of the semi-strapdown homing seeker," Proceedings of the Institution of Mechanical Engineers. Part I: Journal of Systems and Control Engineering, vol. 226, no. 4, pp. 550-562, 2012.

[6] S.-A. Jang, C.-K. Ryoo, K. Choi, and M.-J. Tahk, "Guidance algorithms for tactical missiles with strapdown seeker," in Proceedings of the SICE Annual Conference, pp. 2616-2619, IEEE, Tokyo, Japan, August 2008.

[7] Y. Yifang, Research on Guidance and Control Technology for Strapdown Guided Munition, Beijing Institute of Technology, Beijing, China, 2015.

[8] Z.-J. Fu, W.-D. Xie, and X.-B. Ning, "Adaptive nonlinear tireroad friction force estimation for vehicular systems based on a novel differentiable friction model," Mathematical Problems in Engineering, vol. 2015, Article ID 201062, 7 pages, 2015.

[9] Z. Zhiyong, A Study on Key Measurement and Control Problems of Electro-Optical Stabilization Servo Mechanism, National University of Defense Technology, Changsha, China, 2006.

[10] J. Song, G. Cai, L. Kong, and J. Fan, "Precision analysis of the semi-strapdown homing guided system," Journal of Aerospace Engineering, vol. 27, no. 1, pp. 151-167, 2014.

[11] J. Xu, J. Wang, T. Song, and K.-R. Hu, "A disturbance observerbased inhibition method for disturbance rejection rate of seeker," Acta Armamentarii, vol. 35, no. 11, pp. 1790-1798, 2014.

[12] A. Lawrence, Modern Inertial Technology: Navigation, Guidance, and Control, Springer Science and Business Media, 2012.

[13] R. Yin, R. Wang, X. Y. Zhou, X. Y. Peng, and K. Wang, "Dynamic modeling and nonlinear decoupling control of inertial stabilized platform for aerial remote sensing system," Advanced Materials Research, vol. 898, pp. 807-813, 2014.

[14] S. S. Rao and S. S. Rao, Engineering Optimization: Theory and Practice, John Wiley and Sons, 2009.

[15] Moore and Holly, MATLAB for Engineers, Prentice Hall Press, 2014. 


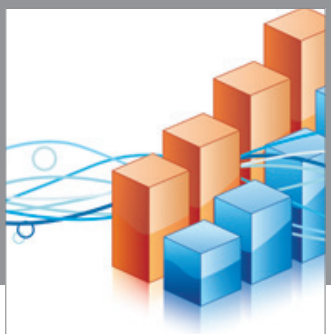

Advances in

Operations Research

vatem alat4

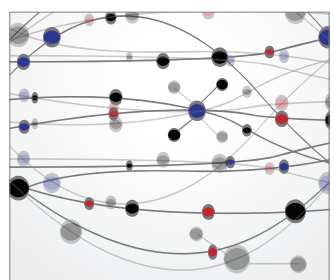

\section{The Scientific} World Journal
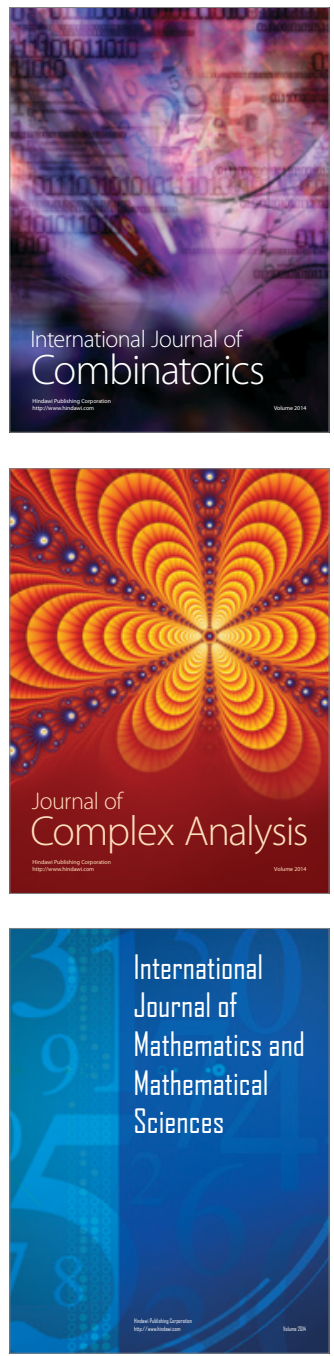
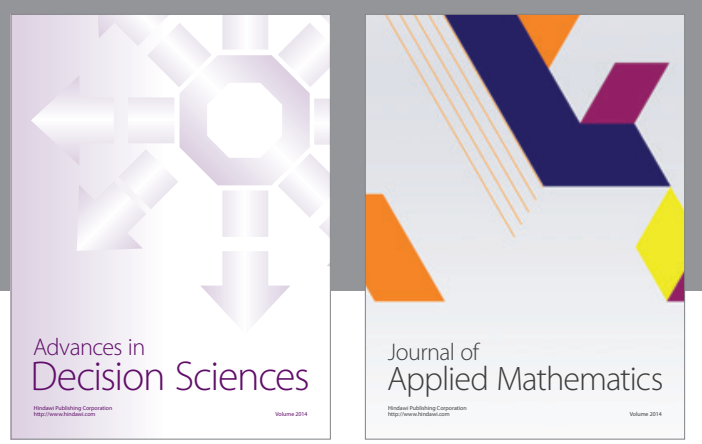

Algebra

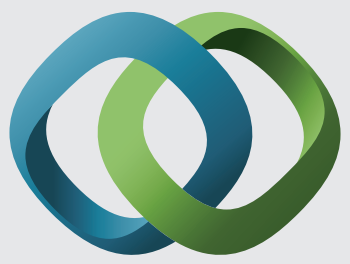

\section{Hindawi}

Submit your manuscripts at

http://www.hindawi.com
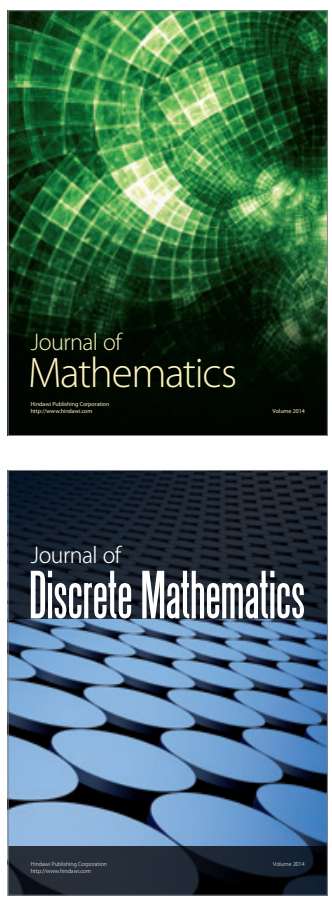

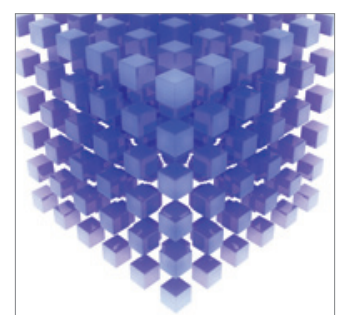

Mathematical Problems in Engineering
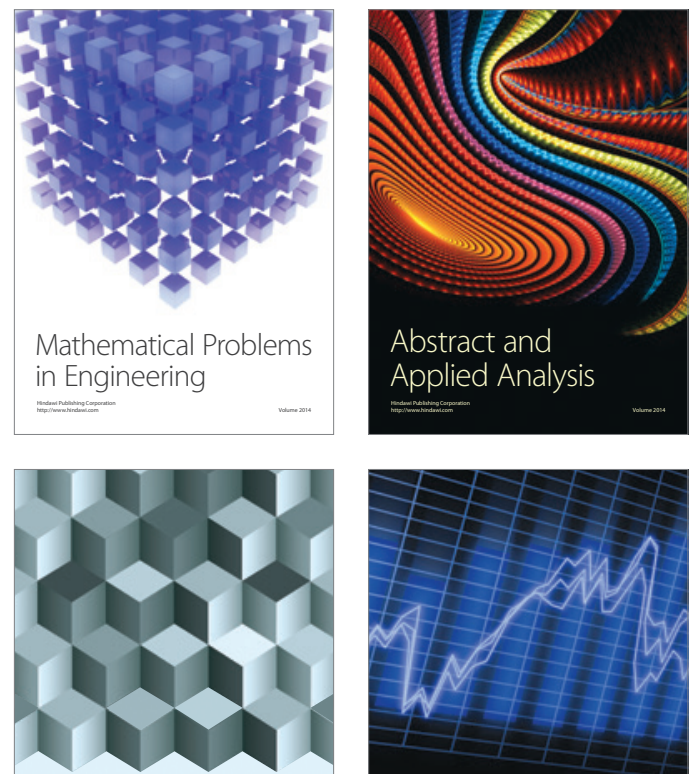

Journal of

Function Spaces

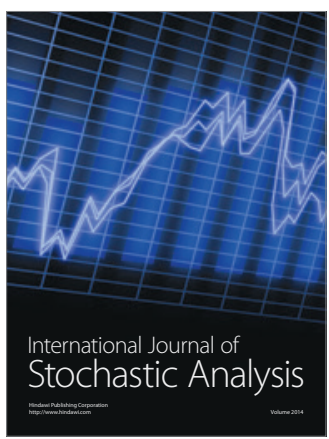

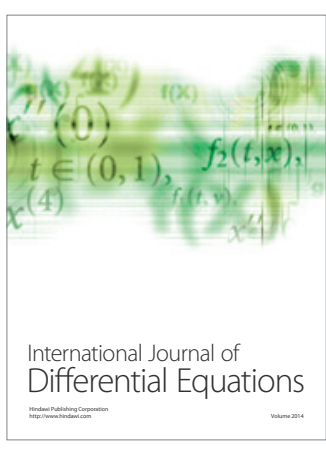
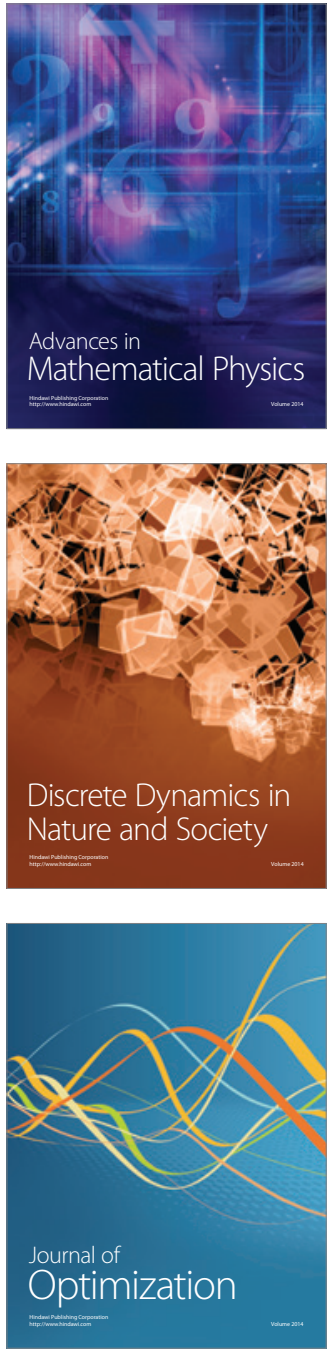